\title{
Morfometría y masa corporal de Strigiformes (Aves) de Argentina
}

\author{
Morphometry and Body Mass of Strigiformes (Birds) from \\ Argentina
}

D.0.I.: https://doi.org/10.30550/j.azl/2018.62.1/2

Ortiz, Diego ${ }^{1,2}$; Capllonch, Patricia ${ }^{2,3}$; Aráoz, Rodrigo ${ }^{2,4}$; Moreno Ten, Thania ${ }^{1,2}$; Zelaya, Josefina ${ }^{1}$; Merino, Víctor Emanuel ${ }^{1}$

1 Centro de Rehabilitación de Aves Rapaces (CeRAR), Reserva Experimental Horco Molle, Facultad de Ciencias Naturales e Instituto Miguel Lillo, Universidad Nacional de Tucumán, Miguel Lillo 205, San Miguel de Tucumán (4000), Tucumán, Argentina.

a Centro Nacional de Anillado de Aves (CENAA), Facultad de Ciencias Naturales e Instituto Miguel Lillo, Universidad Nacional de Tucumán, Miguel Lillo 205, San Miguel de Tucumán (4000), Tucumán, Argentina. Correo electrónico: cenaarg@yahoo.com.ar

3 Cátedra de Biornitología Argentina, Facultad de Ciencias Naturales e Instituto Miguel Lillo, Universidad Nacional de Tucumán, Miguel Lillo 205, San Miguel de Tucumán (4000), Tucumán, Argentina

4 CONICET, Instituto de Ecología Regional (IER), Horco Molle, Facultad de Ciencias Naturales e Instituto Miguel Lillo, Universidad Nacional de Tucumán, Yerba Buena (4007), Tucumán, Argentina.

> Resumen - Presentamos datos de 113 ejemplares vivos de 11 taxones diferentes de rapaces nocturnas de Argentina. La información fue obtenida mediante las campañas de anillado del Centro Nacional de Anillado de Aves (CENAA) y del Centro de Rehabilitación de Aves Rapaces (CeRAR) ambos pertenecientes a la Universidad Nacional de Tucumán. Argentina. Utilizamos redes de niebla durante la noche para capturar las lechuzas durante dichas campañas. En el campo tomamos medidas de longitud total, pico (culmen sin cera y culmen con cera), cuerda alar y masa corporal de cada ave. El objetivo del trabajo fue obtener información biométrica de ejemplares vivos de lechuzas que podrá ser utilizada posteriormente en estudios ecológicos, taxonómicos y de filogenia.

Palabras claves: Tytonidae, Strigidae, lechuzas, biometría.

Abstract - We present data of 113 live specimens of 11 different taxa of night raptors from Argentina. The information was obtained through ringing campaigns of the Centro Nacional de Anillado de Aves (CENAA) and the Centro de Rehabilitación de Aves Rapaces (CeRaR), both belonging to the National University of Tucumán. Argentina. We used mist nests during the night to capture the owls during such campaigns. In the field, we took measures of total length, bill (culmen with wax and culmen without wax), wing chord, and body mass of each bird. The objective of the work was to obtain biometric information of live owl specimens that can be used later in ecological, taxonomic and phylogenetic studies.

Key-words: Tytonidae, Strigidae, owls, biometrics.

> Ref. bibliográfica: Ortiz, Diego; P. Capllonch, R. Aráoz, T. Moreno Ten, J. Zelaya, V. E. Merino (2018). Morfometría y masa corporal de Strigiformes (Aves) de Argentina. Acta zoológica lilloana 62 (1): 10-27.

> Recibido: 13/11/17 - Aceptado: 09/05/18

> URL de la revista: http://actazoologica.lillo.org.ar

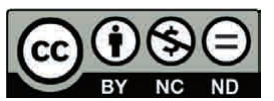




\section{INTRODUCCIÓN}

El objetivo principal de este estudio fue obtener mediciones morfométricas de lechuzas vivas plausibles de ser a su vez comparadas entre grupos o individuos, para explorar los patrones de forma. Este tipo de estudios puede ayudar a comprender el significado biológico de los datos morfológicos y puede proveer información relevante sobre la ecología y la evolución de las aves (Tellería, De La Hera, Pérez-Tris, 2013). Usándose como un índice de las relaciones ecológicas entre especies que coexisten en el mismo hábitat (Ricklefs and Cox, 1977), puede predecir con precisión la ecología de las aves (Botero-Delgadillo, Bayly, 2012). Estudios previos comprobaron, por ejemplo, que la masa corporal varía mucho según el sexo, pero también con la altitud y latitud y entre ecotipos o subespecies (Aráoz, Ortíz, Capllonch, 2016). En Argentina están presentes 20 especies de lechuzas y búhos de cuya biología poco se conoce (Trejo, Bó, Bellocq, López De Casenave, 2007), debido principalmente a las dificultades que plantea la captura y estudio de aves nocturnas. Sin embargo, aún son escasos los estudios morfométricos basados en ejemplares vivos.

Belton (1984) y Dunning (2008) publicaron datos de masa corporal para Sudamérica, pero en Argentina existen pocos estudios de rapaces diurnos y nocturnos que presenten datos morfométricos (Trejo, 2007; Aráoz, et al., 2016). En la mayoría de los casos los datos son de ejemplares taxidermizados de colecciones de museos, que no contienen datos de ejemplares vivos y no contienen datos de masa corporal, los cuales son de gran utilidad, por ejemplo, en los centros de rehabilitación de fauna silvestre, para tener un mayor entendimiento del estado sanitario de los animales ingresados y su posterior rehabilitación. Otras medidas biométricas pueden ser comparadas con poblaciones aisladas de una misma especie y conocer las variaciones morfológicas de los ecotipos (Aráoz et al., 2016). Estas medidas pueden ser correlacionadas con el uso del hábitat entre diferentes especies de una misma zona para explorar aspectos ecológicos.
Dada la escasez de datos morfométricos de lechuzas a partir de ejemplares vivos, el objetivo de este estudio es obtener medidas biométricas y de masa corporal, y la descripción detallada de fases de plumaje de aves vivas siguiendo un protocolo que se aplica en cada uno de los ejemplares analizados, permitiendo así que estos datos sean comparables.

\section{MATERIALES Y MÉTODOS \\ ÁREA DE ESTUDIO}

Los ejemplares de lechuzas fueron obtenidos entre 1987 y 2017 en diferentes localidades de Argentina (Figura 1). SALTA: Bajada de Delfín, $20 \mathrm{~km}$ al oeste de Santa Victoria Este ( $22^{\circ} 162$ S; $62^{\circ} 422$ W) ÿp, Las Mercedes, Ro-

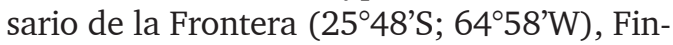

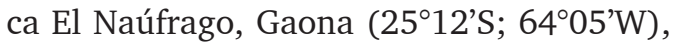
Potrerillos $\left(26^{\circ} 08^{\circ} \mathrm{S} ; 65^{\circ} 46^{\circ} \mathrm{W}\right)$, El Galpón (252 232 S; $\left.64^{\circ} 382 \mathrm{~W}\right)$, Arroyo del Quema-

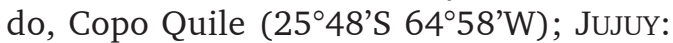

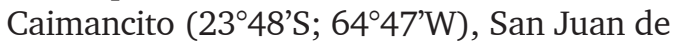

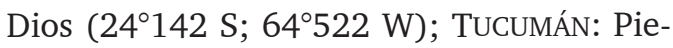
dra Tendida (26⒉ $26^{\prime}$; $\left.64^{\circ} 54^{\prime} \mathrm{W}\right)$, El Bañado, Amaicha del Valle (26 $36^{\circ} \mathrm{S}$; $\left.65^{\circ} 55^{\prime} \mathrm{W}\right)$, Raco (263ㄹ S; $65^{\circ} 262 \mathrm{~W}$ ), El Cadillal (26372 S; $\left.65^{\circ} 122 \mathrm{~W}\right)$, San José de Chasquivil (264'S; 653' 'W), La Ramada (26412 S; $64^{\circ} 562$

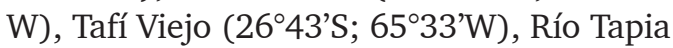
y Ruta Nacional 9 (26 $\left.41^{\prime} ; 6^{\circ} 27^{\prime} \mathrm{W}\right)$, Senda de Velárdez ( $26^{\circ} 43^{\prime} S$; $65^{\circ} 22^{\prime} \mathrm{W}$ ), El Infierni-

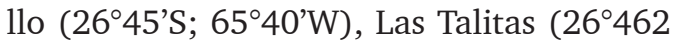
S $65^{\circ} 122 \mathrm{~W}$ ), Yerba Buena (264' $\mathrm{S}$;

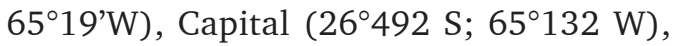
Cruz Alta $\left(26^{\circ} 502 \mathrm{~S}\right.$; $\left.65^{\circ} 092 \mathrm{~W}\right)$, El Manantial $\left(26^{\circ} 512\right.$ S $\left.65^{\circ} 172 \mathrm{~W}\right)$, San Pablo (265'의 $65^{\circ} 18^{\prime} \mathrm{W}$ ), La Reducción ( $26^{\circ} 572 \mathrm{~S}$; $65^{\circ} 212 \mathrm{~W}$ ), Los Nogales (265'ㅇ6 6512'W),

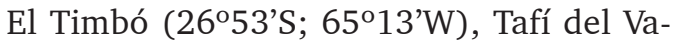

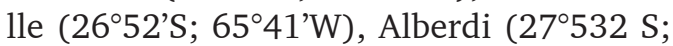

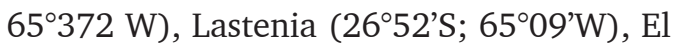
Bracho $\left(26^{\circ} 592 \mathrm{~S} ; 65^{\circ} 102 \mathrm{~W}\right)$, Las Cejas (265'S; 644ㄴ'W), Ranchillos (26562 S; $\left.65^{\circ} 022 \mathrm{~W}\right)$, Leales $\left(27^{\circ} 092 \mathrm{~S} 65^{\circ} 152 \mathrm{~W}\right)$, Cochuna $\left(27^{\circ} 18^{\prime} \mathrm{S} 65^{\circ} 55^{\prime} \mathrm{W}\right)$, Concepción

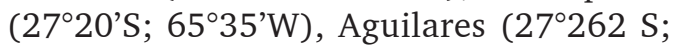
$\left.65^{\circ} 372 \mathrm{~W}\right)$; SANTIAGO DEL Estero: Parque Nacional Copo (255'의 $62^{\circ} 7^{\prime} \mathrm{W}$ ), Santo Do- 
mingo, Río Salado (26²2 S; $\left.63^{\circ} 462 \mathrm{~W}\right)$, Bobadal (264's; 65³3'W), Finca El Duen-

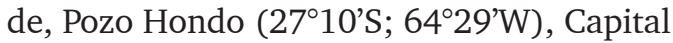

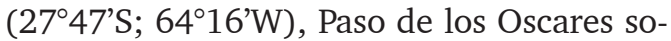
bre Río Utis (2926’ $\left.63^{\circ} 21^{\prime} \mathrm{W}\right)$, Santa Ana,
Sumampa $\left(29^{\circ} 22^{\prime} \mathrm{S} ; 6^{\circ} 28^{\prime} \mathrm{W}\right)$; CATAMARCA: Andalgalá $\left(27^{\circ} 362 \mathrm{~S} ; 66^{\circ} 192 \mathrm{~W}\right)$, Quebrada del Durazno $\left(28^{\circ} 102 \mathrm{~S} ; 5^{\circ} 412 \mathrm{~W}\right)$; CHACO: Las Palmitas $\left(25^{\circ} 32^{\prime} \mathrm{S}, 65^{\circ} 21^{\prime} \mathrm{W}\right)$, Taco Pozo (25362 S; $\left.63^{\circ} 152 \mathrm{~W}\right) \mathrm{ÿp}$; CORRIENTES: Es-

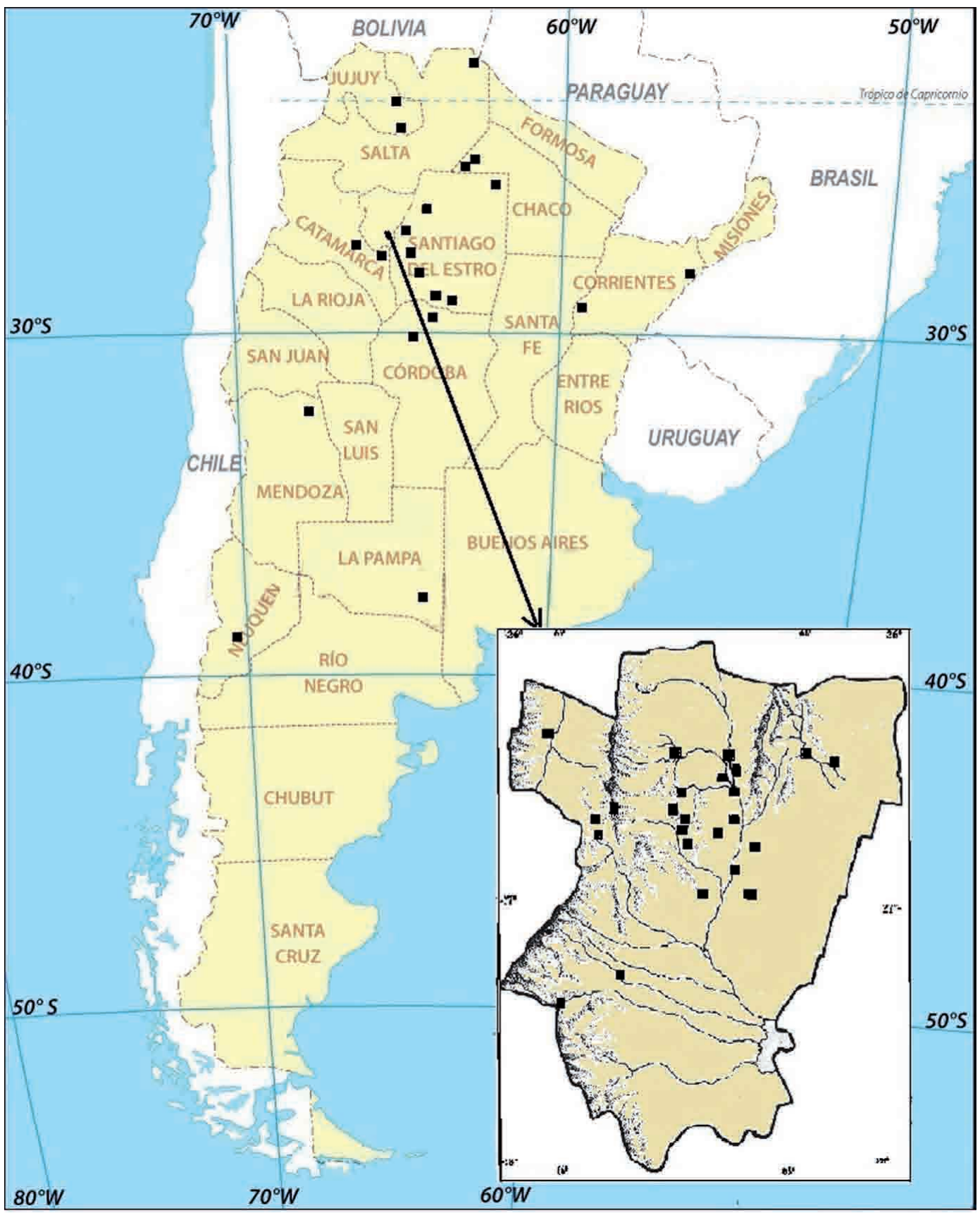

Figura 1. Localidades de procedencia de lechuzas entre los 220 (norte de Salta) y los 40 ㅇe latitud sur (Neuquén) en Argentina que figuran en el texto. Se amplificó el mapa de la pequeña provincia de Tucumán (debajo a la derecha) para facilitar la ubicación de las localidades. 
tancia La Blanca, $17 \mathrm{~km}$ al norte de Santo Tomé ( $28^{\circ} 29^{\prime}$ S; 555' W), Río Santa Lucía, $11 \mathrm{~km} \mathrm{NE}$ de Goya (29032 S; 59 $112 \mathrm{~W}$ ); CóRDobA: Mar Chiquita (30³72 S; $62^{\circ} 332$

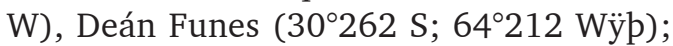
MENDOZA: Telteca $\left(32^{\circ} 232 \mathrm{~S}\right.$; $\left.68^{\circ} 012 \mathrm{~W}\right) \mathrm{ÿp}$;

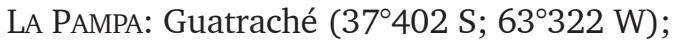
NEUQuÉN: San Martín de los Andes (40¹02 S; $\left.71^{\circ} 212 \mathrm{~W}\right)$.

\section{FUENTE DE DATOS Y MUESTREO}

Los datos fueron obtenidos en campañas de anillado del Centro Nacional de Anillado de Aves (CENAA) en Argentina, en los años 1988 (octubre y noviembre), 1990 (junio), 1991 (julio),1992 (mayo), 1995 (julio), 2003 (septiembre), 2004 (abril, octubre, diciembre), 2005 (octubre, diciembre), 2006 (abril, octubre), 2008 (septiembre, noviembre, diciembre), 2009 (octubre), 2010 (diciembre), 2011 (septiembre), 2012 (abril, septiembre), 2013 (marzo). Otra fuente de datos fue del Centro de Rehabilitación de Aves Rapaces (CeRAR) de la Reserva Experimental de Horco Molle, Facultad de Ciencias Naturales e Instituto Miguel Lillo, Universidad Nacional de Tucumán. Los datos biométricos y especialmente masa corporal son importantes como datos testigos antes de la liberación de aves en cautiverio por lo que el CeRAR lleva un registro de medidas corporales de todas las aves que ingresan a ese centro. Las lechuzas no presentan dimorfismo sexual, por lo que el sexo de algunos individuos fue determinado al hacer necropsias en el CeRAR.

Durante el trabajo de campo operamos redes de niebla de 12 y $9 \mathrm{mt}$ de largo y de 30 a $26 \mathrm{~mm}$ de trama para las especies del género Glaucidium y Megascops, mientras que para las especies de mayor tamaño se utilizaron redes de $9 \mathrm{mt}$ de largo por 2,50 $\mathrm{mt}$ de alto con una trama de tejido de 50 $\mathrm{mm}$ (Ralph, et al., 1996). Las redes fueron colocadas estratégicamente en lugares elegidos según los hábitos que conocíamos de las diferentes especies de lechuzas, lugares al lado de pequeños cursos de agua, áreas abiertas dentro del bosque cerrado, al lado de cuevas o huecos de árboles con murciélagos y en zonas de borde entre el bosque y áreas de pastizales. Fueron colocadas con parantes largos a una altura de tres o cuatro metros, y en algunos casos otras fueron izadas con poleas a alturas de 8 metros en bosques altos (Capllonch, Barquez, Laredo, 1986). En algunos casos se realizaron reproducciones de cantos (playback) para atraer a las aves a las redes. Las redes operaron durante el crepúsculo y la noche, las capturas se obtuvieron entre las 20 hs y las 6 hs del día siguiente.

Tomamos datos morfométricos de longitud total, ala plegada (cuerda alar), cola, (dorsalmente, desde la glándula uropigial a la punta de la cola), pico (culmen con cera y culmen sin cera), tarso y masa corporal (Ralph, et al., 1996). Utilizamos regla metálica con tope para medir longitud total, ala y cola, calibre para longitud de pico y tarso y Pesola(C) de 100, 300 y 1000 gr. para masa corporal, las medidas fueron tomadas en milímetros y el peso en gramos. En todos los casos las aves se marcaron con anillos metálicos con la leyenda «Devuelva Inst. Lillo, Tucumán, Arg».

\section{RESULTADOS}

Obtuvimos los datos de masa corporal y biometría de 113 ejemplares vivos de lechuzas de 11 especies de las Familias Tytonidae y Strigidae. La más abundante en registros fue el Caburé, Glaucidium brasilianum con 28 ejemplares de Tucumán, Jujuy, Salta, Chaco, Santiago del Estero, Córdoba y Mendoza, con capturas entre los $22^{\circ}$ (Salta) y los $37^{\circ}$ (La Pampa) de latitud sur. El lechuzón negruzco Asio stygius es también una especie común, obtuvimos 19 ejemplares, todos de Tucumán, ingresados heridos o como juveniles al CeRAR (Ortiz, et al., 2013; Ortiz, 2016). El Alicucú común Megascops choliba tiene también muchos ejemplares con medidas, 17 ejemplares capturados por el CENAA en Salta, Jujuy, Tucumán, Santiago del Estero y Corrientes.

Encontramos que más de una especie de Megascops puede estar en las mismas par- 
celas de bosque, por ejemplo en Arroyo del Quemado, Copo Quile, Salta, capturamos Megascops hoyi y Megascops choliba a 1200 m.s,n.m en bosque de Chaco Serrano con elementos de Yungas el 23 de agosto y el 13 de octubre de 2005. Es interesante que estas dos especies de tamaño y masa corporal similares estén juntas aunque $M$. choliba, que habita el Chaco, podría ascender altitudinalmente desde los bosques xerófilos chaqueños de la llanura solo durante el invierno a invernar. También capturamos en las mismas parcelas a $M$. choliba uruguaiensis y $M$. atricapillus en Corrientes en Selva Paranaense en galería frente a la Isla de San Mateo, en la ribera oeste del Río Uruguay (28 ${ }^{\circ} 29^{\prime} \mathrm{S}$ 555' W), $17 \mathrm{Km}$ al norte de Santo Tomé (Capllonch, Lobo, Ortiz, Ovejero, 2005). El único ejemplar de $M$. atricapillus capturado el 17 de septiembre de 2003 tiene medidas un poco más grandes que $M$. choliba (Tabla 1). Los ejemplares de M. choliba wetmorei capturados en varias provincias argentinas presentaron una gran variación de masa corporal. M. choliba wetmorei (Brodkorb, 1937), se distribuye en el Chaco de Paraguay y Argentina, hasta el sur de Mendoza y noreste de Buenos Aires. M. c. uruguaiensis (Hekstra, 1982), se encuentra en el sureste de Brasil (Santa Catarina, Río Grande do Sul), el noreste de Argentina y todo Uruguay.

Los ejemplares adultos más australes Glaucidium brasilianum de Córdoba (Mar Chiquita y Deán Funes) y La Pampa (Guatraché), son mucho más grandes, poseen entre 80 y 87 gr, que los del resto del norte de Argentina entre 45 y 66 gr. (Tabla 1). Esta especie es polimórfica en plumaje, presentando gran plasticidad fenotípica, esto es que individuos con el mismo genotipo pueden presentar diferencias fenotípicas (West-Eberhart, 1989). Hemos encontrado tres fases de coloración del plumaje: gris, parda y canela (Figura 2) en la misma localidad en Taco Pozo, provincia de Chaco, donde fueron abundantes en agosto de 2007. Todas tienen un disco facial blancuzco, cejas claras marcadas, iris amarillo, pupilas negras, pico amarillo y tarsos y dedos amarillos. Los tarsos son poco emplumados (comparados con otras especies de lechuzas) hasta los talones y los dedos sin plumas aunque con cerdas y con afiladas garras. El plumaje de la cabeza varía del pardo (café o chocolate) al canela intenso (anaranjado en algunos individuos) y hay una fase gris (gris claro o gris oscuro), presenta gran cantidad de rayas o estrías y motas blancas. En la nuca tienen una mancha negra a cada lado de la parte posterior del cuello comúnmente llamadas «ojos falsos». El plumaje general del dorso presenta rayas que pueden ser grises, café, o canelas intensas según las fases. Las cobertoras alares y plumas del vuelo están barradas de blanco. La cola es obscura con 5 u 8 barras claras. La fase rojiza presenta el mismo patrón de coloración pero con tonos rojizos o café-anaranjados. Los juveniles son similares a los adultos pero con patrones de coloración clara, no tienen un desarrollo completo de las estrías de la cabeza y presentan una coloración salmón rosada en las cobertoras internas. En la fase canela de un ejemplar de Las Palmitas, Taco Pozo, Chaco (Figura 2, abajo derecha) presentaba cola rufa, dorso pardo claro, ala canela, cabeza con estrías posteriores amarillas, iris y pico amarillos. En esta localidad capturamos las tres fases juntas en la misma época.

Los individuos de Asio stygius, todos adultos, también poseen una gran variación en cuanto a masa corporal, entre 335 y 700 gr (peso promedio 511 gr), también en longitud total y cuerda alar. Casi todos los ejemplares de A. stygius provienen de diferentes localidades boscosas de Yungas, Chaco y zonas urbanas arboladas (parques y jardines) de la provincia de Tucumán (Figura 3). Los ejemplares de Asio clamator, similares en peso promedio que stygius provienen en cambio de áreas urbanas y áreas cultivadas arboladas de Tucumán y menor variación de masa corporal, entre 440 y 590 gr. Son las lechuzas de mayor tamaño entre las que citamos en este trabajo. 


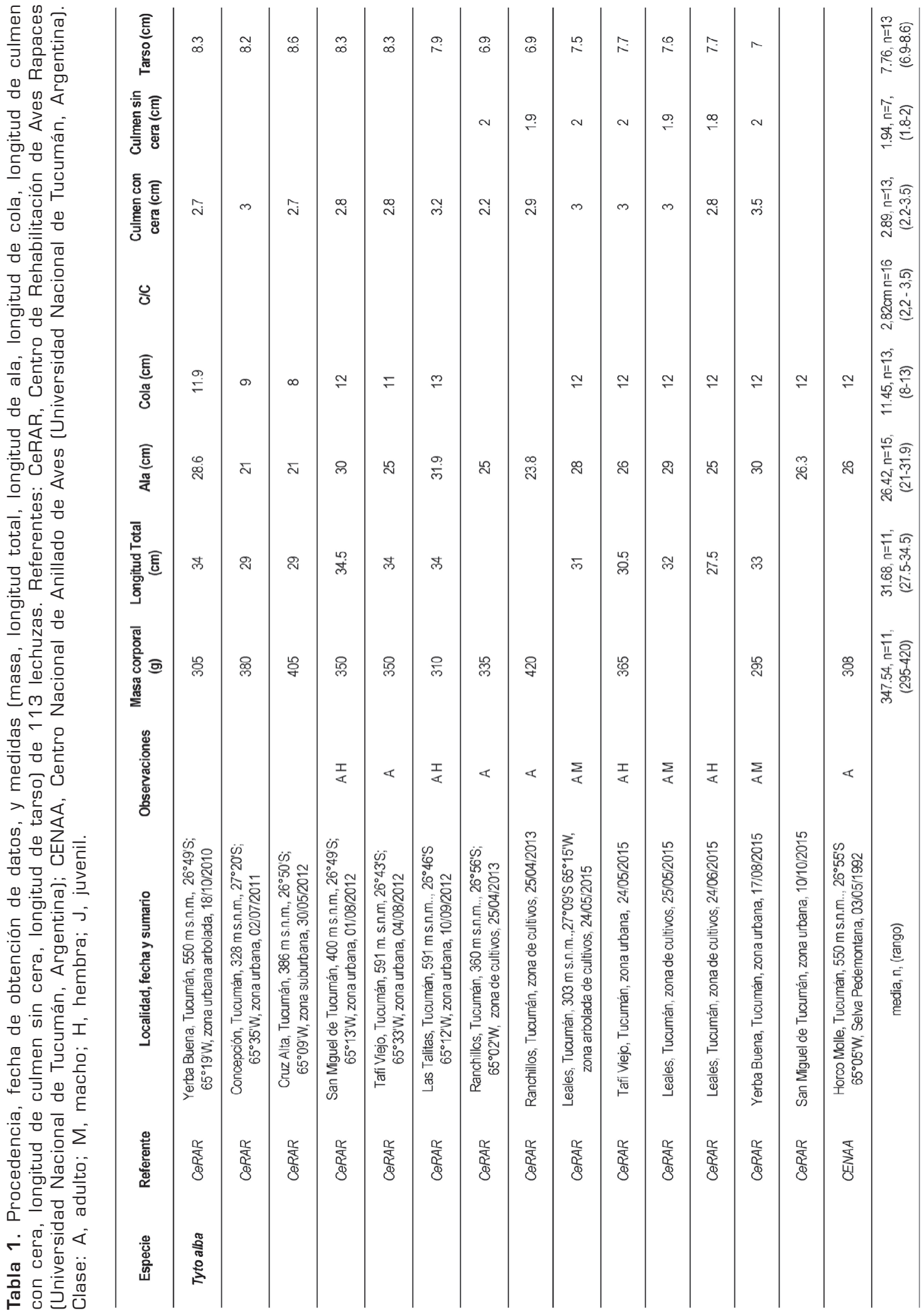




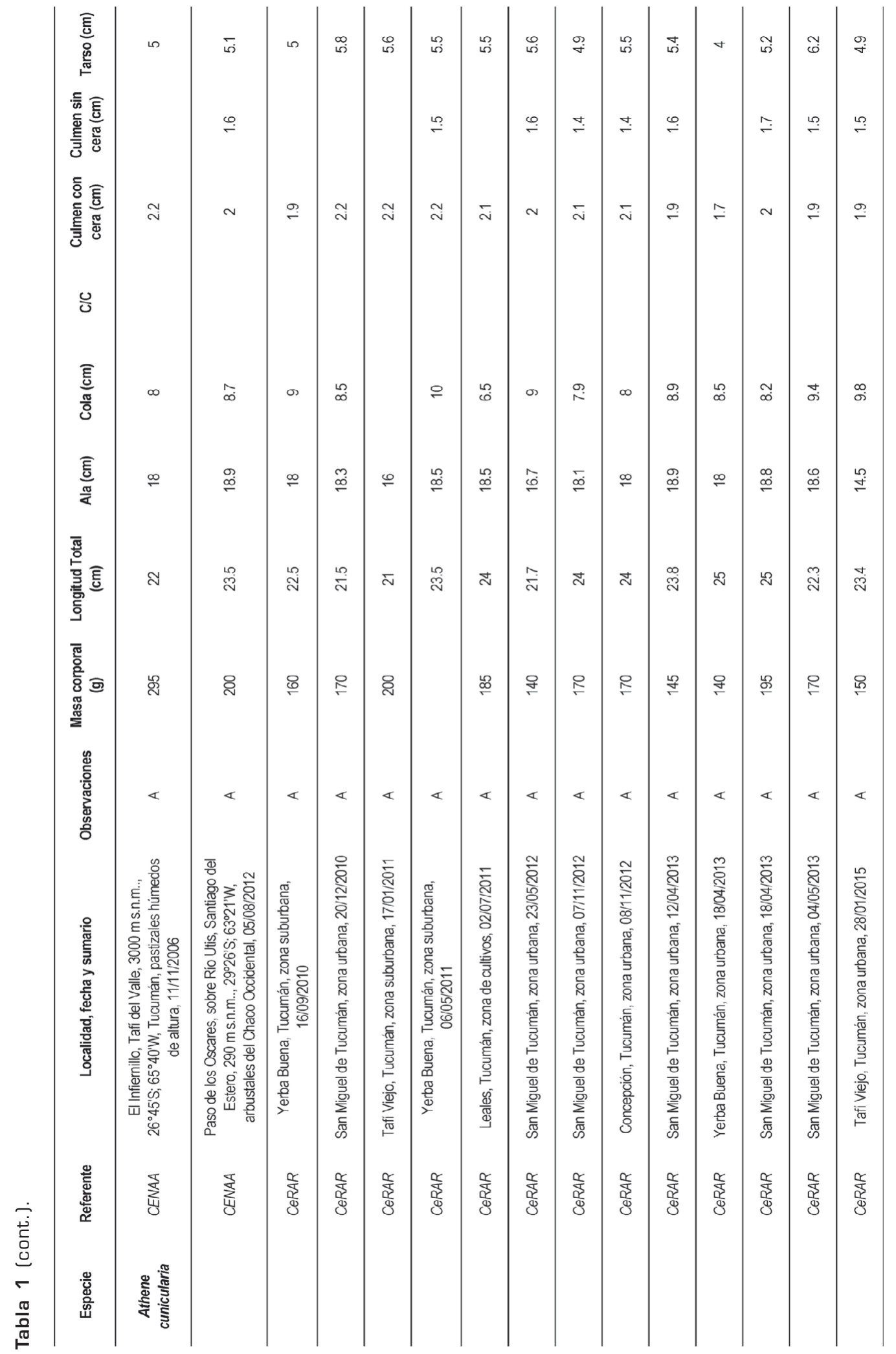




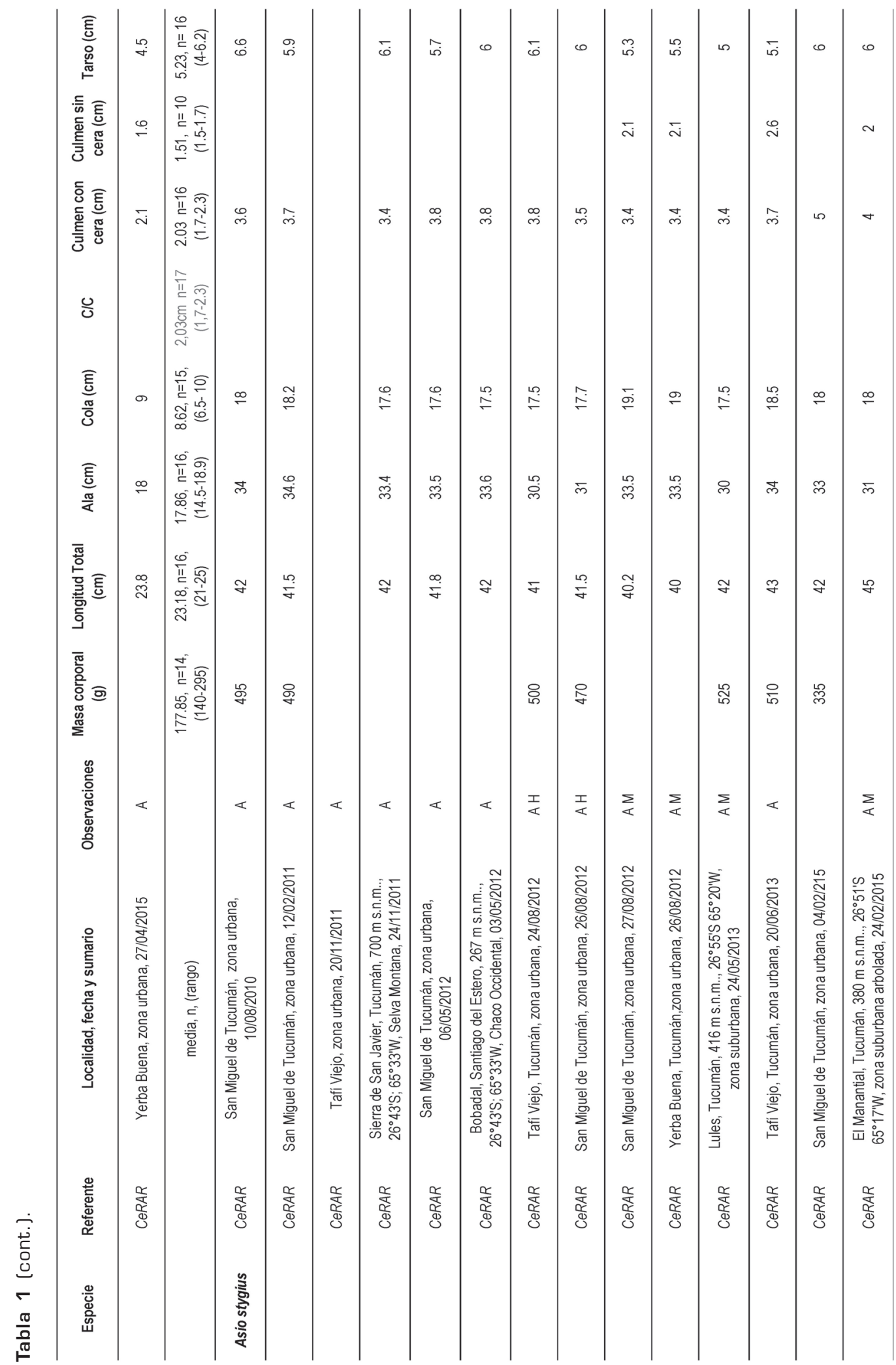




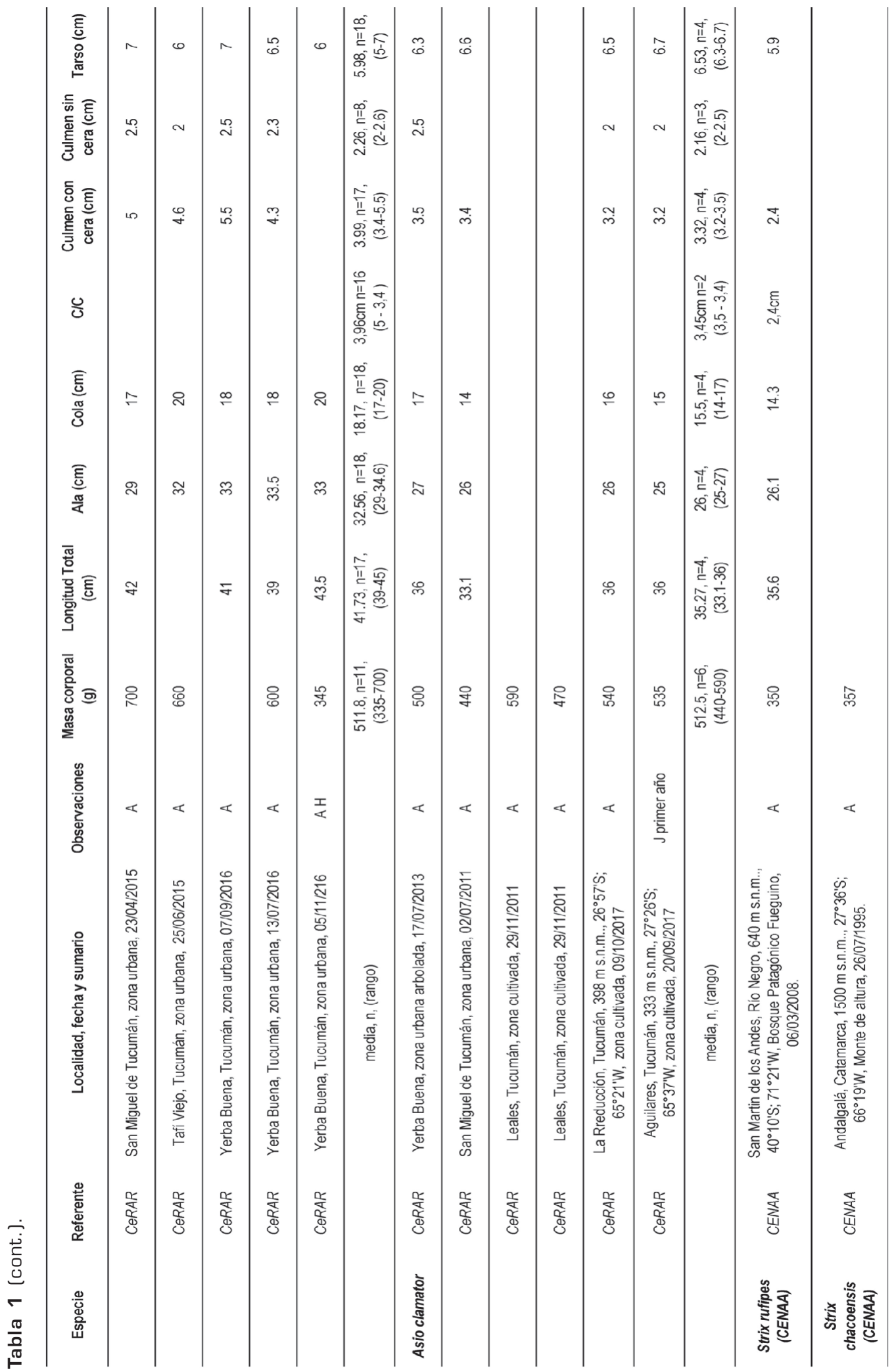




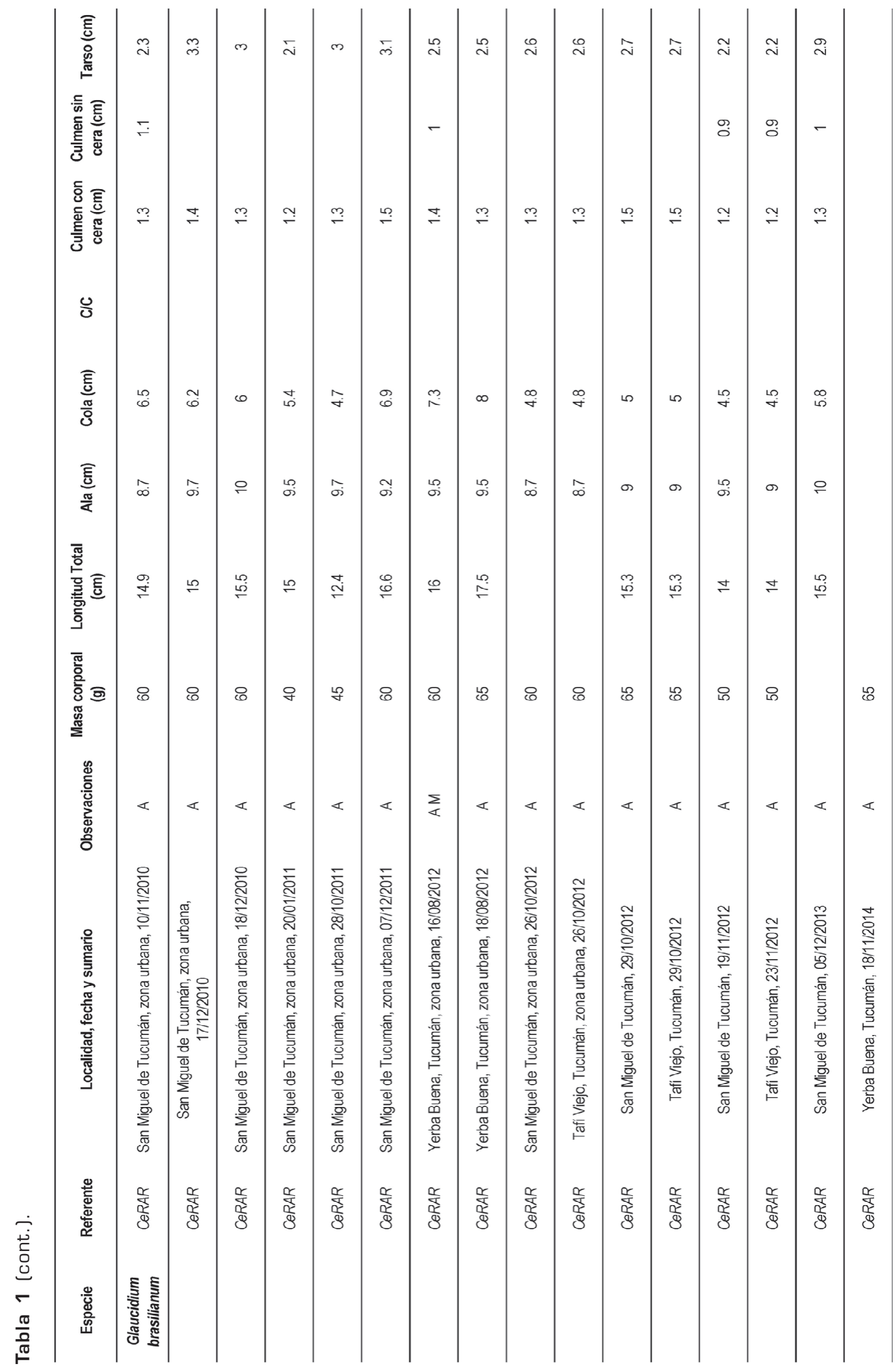




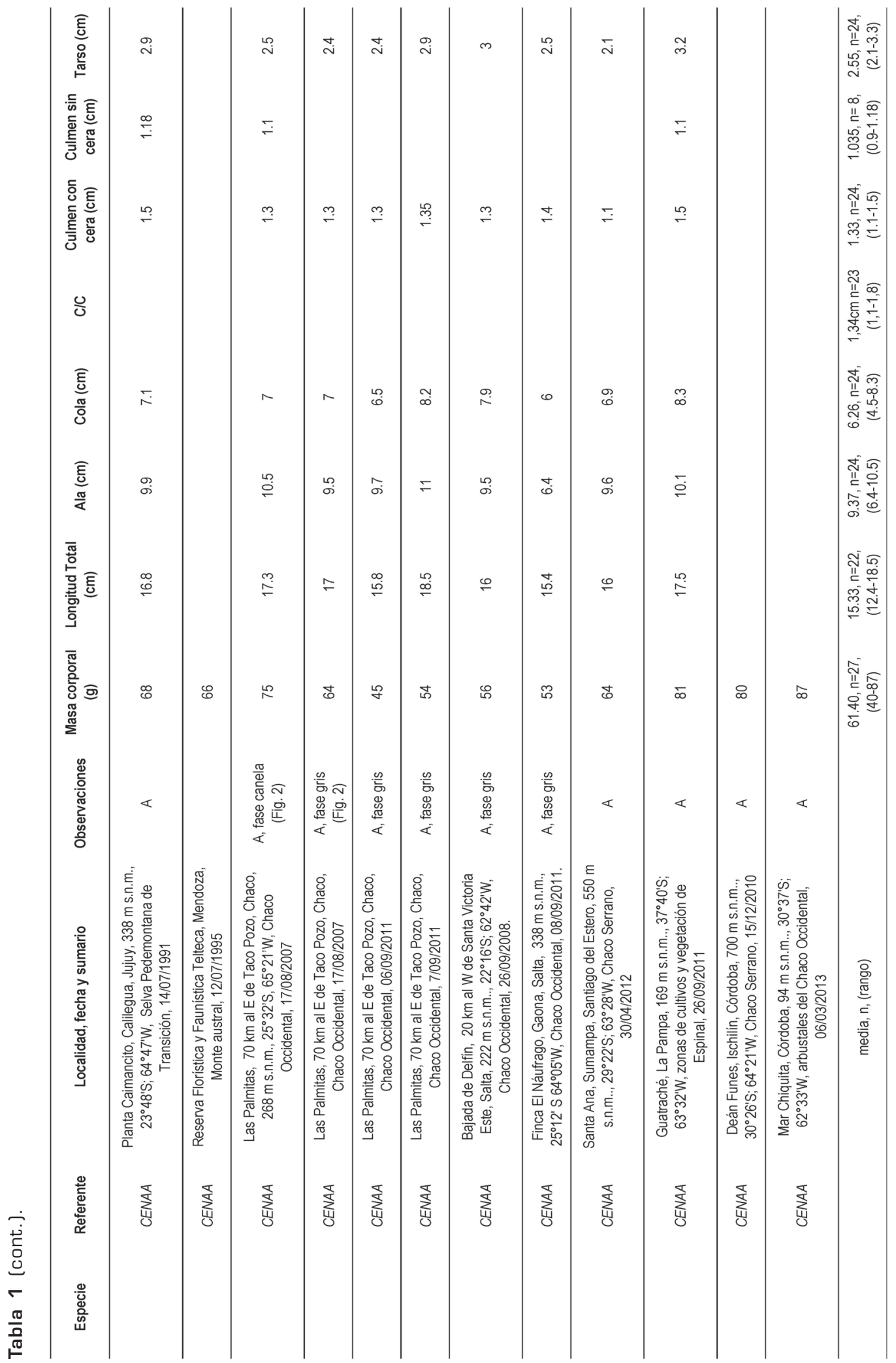




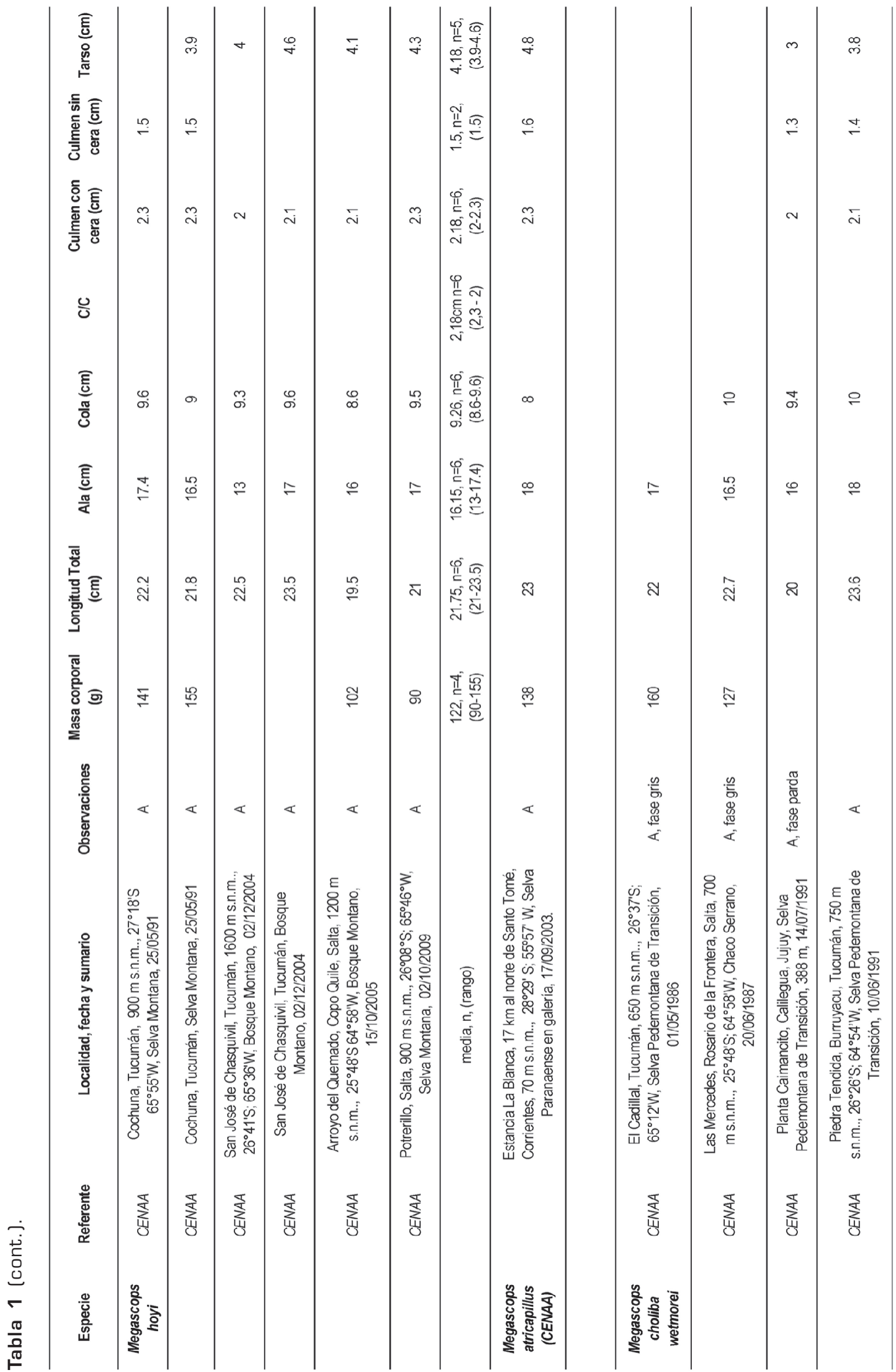




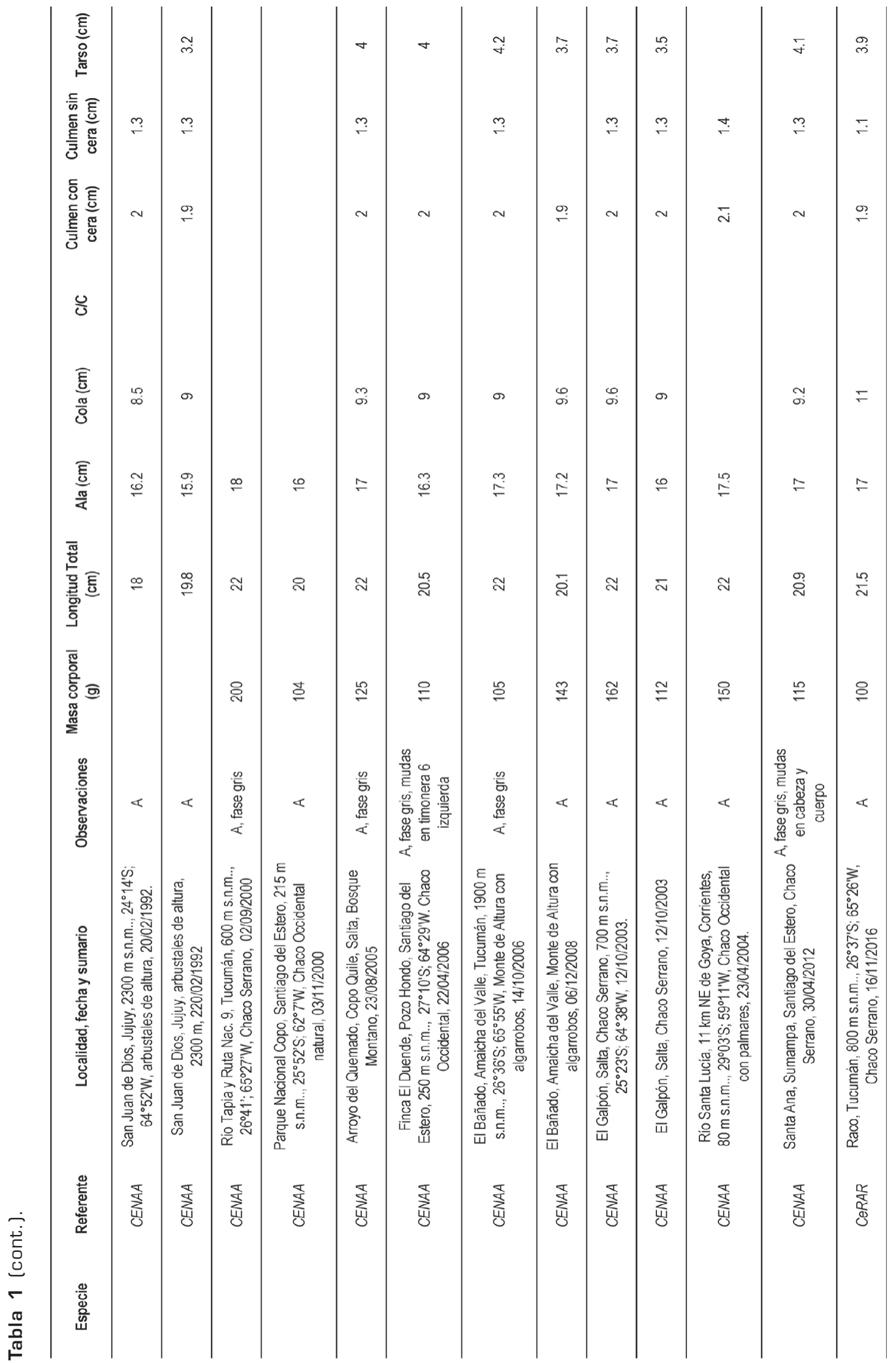




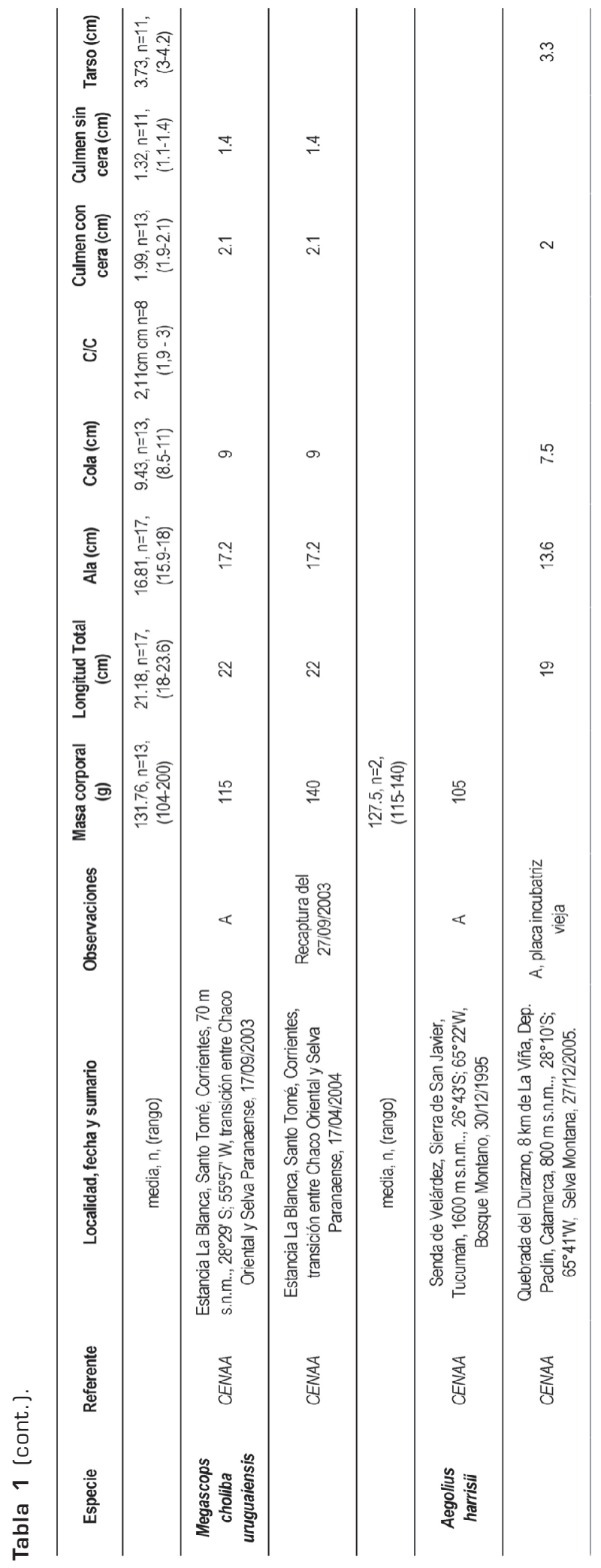



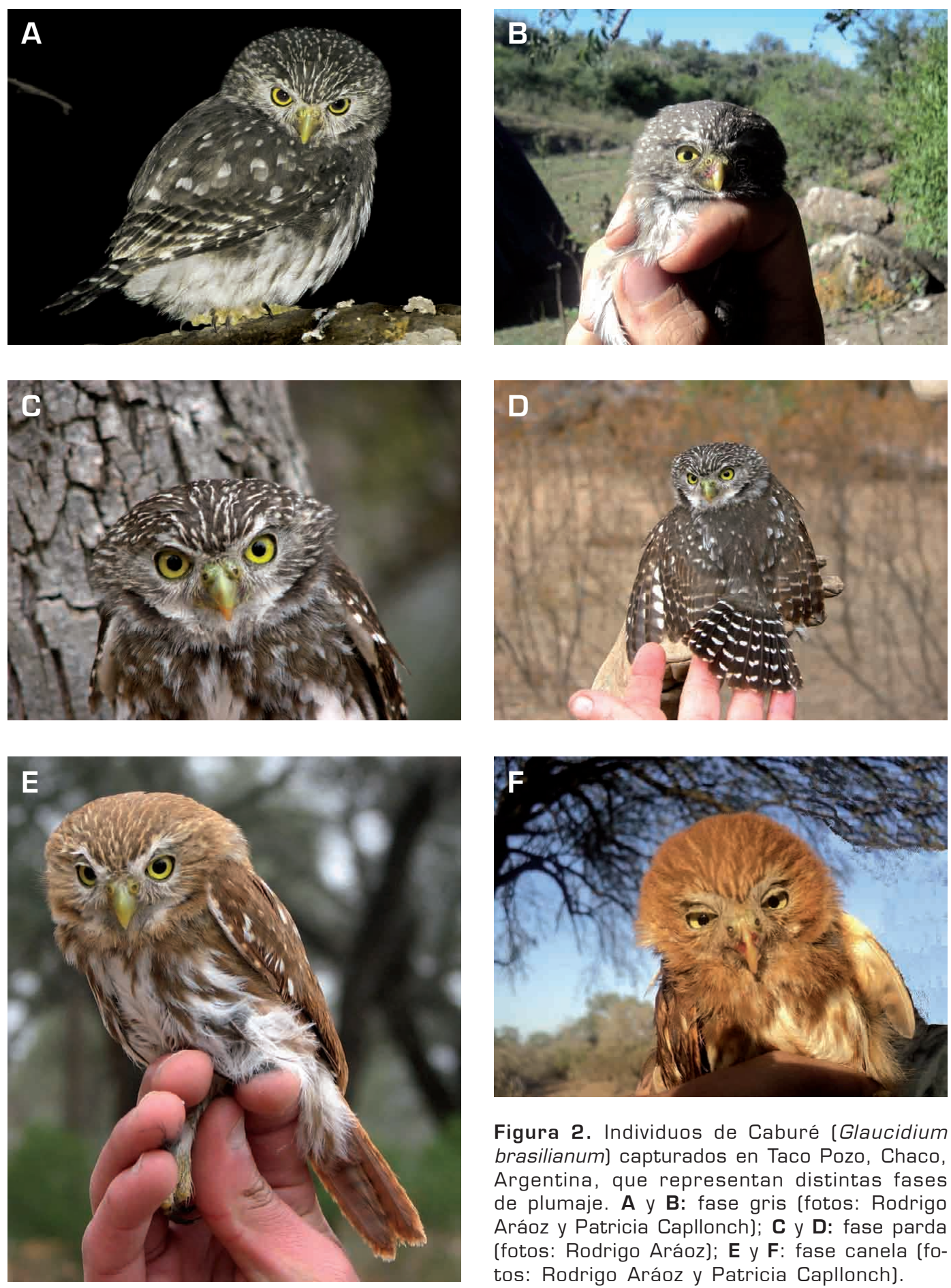

Figura 2. Individuos de Caburé (Glaucidium brasilianum] capturados en Taco Pozo, Chaco, Argentina, que representan distintas fases de plumaje. A y $\mathbf{B}$ : fase gris (fotos: Rodrigo Aráoz y Patricia Capllonch); C y D: fase parda (fotos: Rodrigo Aráoz); E y F: fase canela (fotos: Rodrigo Aráoz y Patricia Capllonch). 


\section{DISCUSIÓN}

Dos puntos de interés presentamos en este trabajo. Por un lado, prácticamente no existen estudios sobre morfometría de rapaces, especialmente nocturnas en Argentina, aunque sí numerosos estudios ecológicos, demográficos y de comportamiento (Salvador, 1981; Morici y Massoia, 1998; Blendinger, Capllonch, Álvarez, 2004; Trejo, et al., 2007; Ortiz, Barrionuevo, Capllonch, Julio, Aráoz, 2009; Trejo y Ojeda, 2015). Por otro lado, en la mayoría de los casos estas aves han sido estudiadas principalmente en áreas abiertas y no en el interior de bosques (Trejo y Ojeda, 2015). Este trabajo aporta datos inéditos sobre la morfometría de lechuzas de selvas y bosques del norte de Argentina. La medida morfométrica que mostró mayor variación entre individuos de una misma especie y de un mismo género fue la masa corporal.

Las capturas muestran que existe una frecuente relación de coexistencia entre las especies del género Megascops, tanto en las zonas pedemontanas del noroeste de Argentina, como en sectores de la Selva Paranaense. En el noroeste se determinó que los ejemplares de Megascops choliba wetmorei, de ambientes chaqueños y pedemontanos, tienen una mayor masa corporal que Megascops hoyi, que habita las selvas montanas y pedemontes. Es muy probable que esta variación morfología influya en la ecología trófica de ambas especies en esta franja de intersección entre el chaco y la selva. Aunque su dieta sería similar (insectos, reptiles, roedores y aves) (Morici y Massoia, 1998; Ortiz, et al., 2009), no existen estudios concretos de la alimentación de ambas especies, para poder relacionarlos con la morfometría. Mientras que en la selva paranaense no se pudo determinar esta relación de masa corporal entre Megascops atricapillus y Megascops choliba uruguaiensis, por insuficiencia de datos.

La gran variación de la masa corporal entre 45 a $87 \mathrm{gr}$. de los individuos de Glaucidium brasilianum, provenientes del sur en relación a los del norte de Argentina, podría estar relacionada a la diferenciación de dos ecotipos, y que en nuestras capturas no han sido tenidos en cuenta. Este género al parecer, es muy variable y se encuentran varias especies enmascaradas, que sólo son diferenciadas por el canto (Straneck, Ridgely, Rodríguez Mata, 1987; Garrido, 2002). Los análisis morfológicos como los de este trabajo podrían aportar un mayor conocimiento de las diferencias entre las distintas poblaciones de especies de este género.

En relación al género Asio, la especie stygius fue la de mayor ingreso en el CeRAR, situación que preocupa ya que un número elevado de estos animales tenían lesiones producidas por el hombre, heridas por hondas y balas. Los registros provienen en su mayoría de zonas urbanas, como parques dentro de la ciudad de San Miguel de Tucumán, mostrando una tolerancia a la presencia humana y una habituación a vivir en las ciudades, algo prometedor para la especie si se toman los recaudos necesarios para su conservación.

Asio clamator ingresó en mucho menor proporción al CeRAR y los ambientes de donde provienen los registros fueron áreas urbanas pequeñas y menos densamente pobladas y rodeadas por arboledas y grandes áreas de cultivos y ganaderas. Si bien hay diferencias entre masa corporal entre estas dos especies del genero Asio, ambas no utilizarían los mismos habientes y tal vez utilicen recursos alimenticios diferentes.

\section{AGRADECIMIENTOS}

Agradecemos a los numerosos ayudantes de campo del CENAA que colaboraron en las campañas de anillado. Agradecemos también a la Facultad de Ciencias Naturales e Instituto Miguel Lillo, Universidad Nacional de Tucumán y a la Reserva Experimental Horco Molle y al Centro de Rehabilitación de Aves Rapaces (CeRAR) por el espacio y apoyo económico brindado para el mantenimiento de las lechuzas en rehabilitación. Agradecemos a IUCN (The International Union for Conservation of Nature and Natural Resources), Administración de Parques Nacionales y Reservas Provinciales por las autorizaciones y permisos de trabajos de rehabilitación y de captura. 


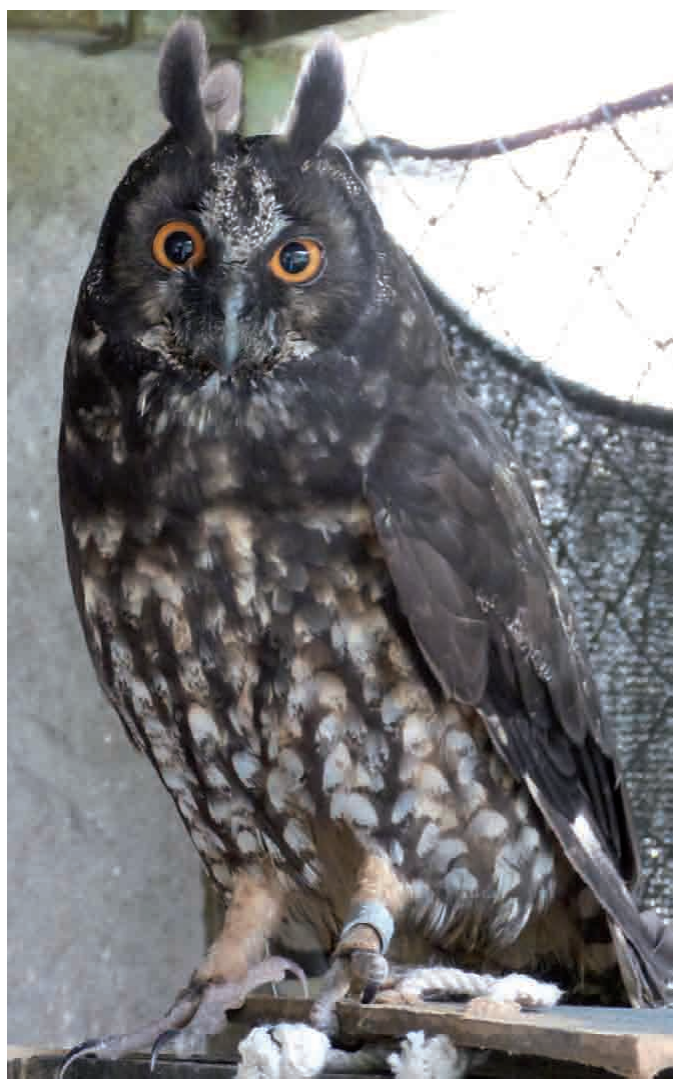

FINANCIAMIENTO

Los viajes del CENAA se realizaron con la ayuda de vehículos, combustibles y viáticos para choferes provistos por la Facultad de Ciencias Naturales e Instituto Miguel Lillo, Universidad Nacional de Tucumán. Los anillos que se utilizaron para las aves fueron financiados por la Reserva de Horco Molle y fondos privados y enviados por Aves Argentinas. El CeRAR es parte de la Reserva de Horco Molle y sus gastos de funcionamiento están incluidos dentro de su presupuesto.

\section{PARTICIPACIÓN}

Diego Ortiz, Patricia Capllonch y Rodrigo Aráoz fueron responsables de la captura de ejemplares por medio de redes, los dos primeros autores redactaron el manuscrito y Thania Moreno Ten, Josefina Zelaya y Víctor Emanuel Merino midieron y pesaron los ejemplares del CeRAR.

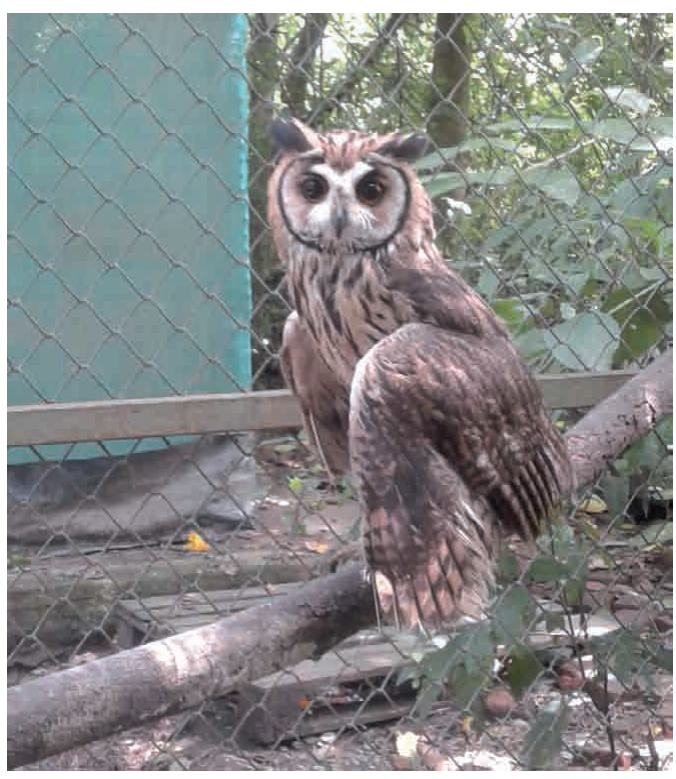

Figura 3. A la izquierda ejemplar de Lechuzón negruzco Asio stygius de San Miguel de Tucumán; a la derecha ejemplar de Lechuzón orejudo Asio clamator de La Reducción, Tucumán, ingresados al CeRAR de la Reserva de Horco Molle, Universidad Nacional de Tucumán para su rehabilitación y liberación (fotos: Diego Ortiz).

\section{CONFLICTOS DE INTERÉS}

Declaramos explícitamente que no existen conflictos de interés entre autores o con terceros.

\section{LITERATURA CITADA}

Aráoz R., Ortiz D., Capllonch P. (2016). Biometrics and body masses of some birds of prey of Argentina. Revista Brasileira de Ornitología, 24 (4), 344-348.

Belton W. (1984). Birds of Rio Grande do Sul, Brazil. Bulletin of the American Museum of Natural History, 178, 369-631.

Blendinger P., Capllonch P. y Álvarez E. (2004). Abundance and distribution of raptors in the Sierra de San Javier Biological Park, Northwestern Argentina. Ornitología Neotropical, 15, 501-512.

Botero-Delgadillo E., Bayly N.J. (2012). Does morphology predict behavior? Correspondence between behavioral and morphometric data in a Tyrant-ûycatcher (Tyrannidae) assemblage in the Santa Marta 
Mountains, Colombia. Journal Field Ornithol, 83[4], 329-342.

Capllonch P., Barquez R.M. y Laredo C.D. (1986). Instrucciones para el anillado. Boletín informativo $\mathrm{N}^{0} 1$. Universidad Nacional de Tucumán. Facultad de Ciencias Naturales e Instituto Miguel Lillo.

Capllonch P., Lobo R., Ortiz D., Ovejero R. [2005). La avifauna de la selva en galería en el noreste de Corrientes, Argentina: Biodiversidad, patrones de distribución y migración. Insugeo, Miscelánea, 14, 361-376. Tucumán.

Dunning Jr, J.B. (2008). CRC handbook of avian body masses, 2nd edn. Boca Raton, CRC Press.

Garrido, 0. H. (2002). Subespecie nueva de Sijú Platanero [Glaucidium siju) para Cuba (Aves: Strigidae), con comentarios sobre otras especies de la familia. SOLENODON, 2, 45-52

Morici A., Massoia E. (1998). Análisis de regurgitados de Otus, choliba choliba (vieiuot, 1817), de la granja -17 de abril- pdo. de Gral. Rodríguez, Buenos Aires. APROMA Boletín Científico. Año $\mathrm{XI}, \mathrm{N}^{\circ} 34$

Ortiz D., Barrionuevo C., Capllonch P., Julio L., Aráoz R. (2009). Acerca de la distribución e historia natural del Alilicucu Yungueño (Megascops hoyi) en el noroeste argentino. Nuestras Aves, 54, 11-14.

Ortiz, D., Mamani J., Moreno Ten T., Jorgieff B., Quiroga O., Barboza E., Alderete C y Aveldaño S. (2013). Rehabilitación y liberación de aves, la importancia del anillado (el caso de un lechuzón orejudo]. Biológica, Revista de Naturaleza Conservación y Sociedad. № 16 (126127). Museo Prov. Cs. Nat. Florentino Ameghino.

Ortiz, D. (2016). Recaptura de un Lechuzón negruzco (Asio stygius), Rehabilitado y liberado por el centro de rehabilitación de aves rapaces (CeRAR], de la Reserva Experimental Horco Molle, Tucumán, Argentina. EcoRegistros Revista, 6 (7), 24-26.
Ralph C.J.; Geupel G.R., Pyle P., Martin T.E., Desante D., Milá B. (1996). Manual de métodos de campo para el monitoreo de aves terrestres. Gen. Tech. Rep. PSWGTR- 159. Albany, CA: Pacific Southwest Research Station, Forest Service, U.S. Department of Agriculture, 46.

Ricklefs R. E., Cox G. W. (1977). Morphological similarity and ecological overlap among passerine birds on St. Kitts, British West Indies. Oikos, 29, 60-66.

Salvador, S.A. (1981). Datos de nidificación de Asio flammeus suinda (Vieillot): (Aves: Strigidae). Historia Natural, 2, 49-52.

Straneck, R., Ridgely, R.S., Rodríguez Mata, J. (1987). Dos nuevas lechuzas para la Argentina: Caburé Andino Glaucidium jardini y Lechucita Vermiculada Otus guatemalae (Aves, Strigidae). Comunicaciones del Museo Argentino de Ciencias Naturales e Instituto Nacional de Investigaciones de las Ciencias Naturales, 4, 137-139.

Tellería J.L., De La Hera I., Pérez-Tris J. (2013). Morphological variation as a tool for monitoring bird populations: a review. Annual Review of Ecology and Systematics, 20, 249-278.

Trejo A. (2007). Bibliografía comentada sobre aves rapaces de Argentina. Hornero, 22, 185-217.

Trejo A., Bó M.S., Bellocq M.I., López De Casenave J. (2007). Ecología y conservación de aves rapaces en Argentina. Hornero, 22, 81-83.

Trejo A., Ojeda V. (2015). Aportes desde la vertiente argentina al conocimiento de las aves rapaces del bosque templado austral. Boletín Chileno de Ornitología, 21 (1-2), 15-28.

West-Eberhart M. J. (1989). Phenotypic plasticity and the origins of diversity. Annual Review of Ecology and Systematics, 20, 249-278. 